\title{
707 IL12 FC-FUSIONS ENGINEERED FOR REDUCED POTENCY AND EXTENDED HALF-LIFE EXHIBIT STRONG ANTI- TUMOR ACTIVITY AND IMPROVED THERAPEUTIC INDEX COMPARED TO WILD-TYPE IL12 AGENTS
}

${ }^{1}$ Matthew Bernett*, ${ }^{1} \mathrm{Ke}$ Liu, ${ }^{1}$ Christine Bonzon, ${ }^{1}$ Michael Hackett, ${ }^{1}$ Katrina Bykova, ${ }^{1}$ Rumana Rashid, 'Nicole Rodriguez, ${ }^{1}$ Nargess Hassanzadeh-Kiabi, 'Connie Ardila, ${ }^{1}$ Norman Barlow, ${ }^{1}$ Irene Leung, ${ }^{1}$ Hanh Nguyen, ${ }^{1}$ Araz Eivazi, ${ }^{1}$ Kendra Avery, ${ }^{2}$ Rajat Varma, ${ }^{1}$ Umesh Muchhal, ${ }^{1} J o h n$ Desjarlais. 'Xencor, Inc., MONROVIA, CA, United States; ${ }^{2}$ Independent Consultant, Monrovia, CA, United States

Background Interleukin-12 (IL12) is a proinflammatory cytokine that induces differentiation of Th1 cells and increased cytotoxicity of T and NK cells. Stimulation by IL12 leads to production of IFN $\gamma$ and an inflammatory tumor microenvironment critical for anti-tumor responses. Studies in mice revealed IL12 can dramatically shrink syngeneic tumors, however human clinical studies resulted in severe toxicity and a small therapeutic window, limiting response rates. Prior work at Xencor demonstrated that reduced-potency IL15/IL15R $\alpha$-Fc fusion proteins exhibited superior therapeutic index (TI) in non-human primates (NHP) by reducing receptor-mediated clearance. Applying similar principles to IL12, we created IL12 heterodimeric Fc-fusions (IL12-Fc) with reduced potency to improve TI.

Methods IL12 is a heterodimer of two subunits, so we engineered IL12-Fc fusions by fusing the IL12p35 subunit to one side of a heterodimeric (and inactive) Fc domain, and IL12p40 to the other side. These Fc-fusions were tuned for optimal activity by introducing amino acid substitutions at putative receptor-interface positions and screening for reductions of in vitro potency. In vitro activity was assessed on human PBMCs by measuring signaling in a STAT4 phosphorylation assay and IFN $\gamma$ production in a mixed-lymphocyte reaction (MLR). In vivo anti-tumor activity of human IL12-Fc was assessed in huPBMC-NSG-DKO and huCD34+ MCF7 xenograft models. Surrogate mouse potency-reduced IL12-Fc were evaluated in syngeneic tumor models. Tolerability and pharmacodynamic activity were assessed in NHP.

Results An IL12-Fc potency series was created, and variants had up to a 10,000-fold reduction in STAT4 signaling and IFN $\gamma$ production in an MLR assay compared to wild-type IL12-Fc. Anti-tumor activity was achieved with potencyreduced IL12-Fc as single-agents and in combination with anti-PD1, with weaker variants maintaining anti-tumor activity at higher dose levels. Analysis of peripheral lymphocytes indicated increased numbers of $\mathrm{T}$ and NK cells as well as activation of $\mathrm{CD} 8+\mathrm{T}$ cells. Increased expression of immune checkpoints including PD1 was also observed. Analysis of serum indicated up to 200 -fold increases in IFN $\gamma$ levels. Surrogate potency-reduced IL12-Fc had improved tolerability and greater selectivity of IFN $\gamma$ production in tumors compared to spleen and less production of IL10 compared to wild-type IL12-Fc. In NHP, potency-reduced IL12-Fc had superior exposure with slower, more sustained accumulation of IFN $\gamma$ and IP10, and a more gradual dose-dependent peak response, as well as more sustained margination of $\mathrm{T}$ and NK cells compared to wild-type IL12-Fc.

Conclusions Potency-reduced IL12-Fc retain strong anti-tumor activity, while potentially overcoming safety and tolerability issues related to narrow TI associated with wild-type IL12 or IL12-Fc agents.

http://dx.doi.org/10.1136/jitc-2021-SITC2021.707 